\title{
Evaluation of Candidate Photomultiplier Tubes for the Upgrade of the CDF End Plug Calorimeter
}

\author{
W. Koska, S. W. Delchamps, J. Freeman, W. Kinney, D. Lewis, P. Limon and J. Strait \\ Fermi National Accelerator Laboratory \\ P.O. Box 500, Batavia, Illinois 60510
}

\author{
I. Fiori \\ University of Bologna \\ Bologna, Italy \\ M. Gallinaro \\ University of Padova \\ Padova, Italy \\ Q. Shen \\ Purdue University \\ West Lafayette, Indiana 47904
}

January 1994

Presented at the Workshop on Scintillating Fiber Detectors,

University of Notre Dame, South Bend, Indiana, October 24-28, 1993 


\section{Disclaimer}

This report was prepared as an account of work sponsored by an agency of the United States Government. Neither the United States Government nor any agency thereof, nor any of their employees, makes any warranty, express or implied, or assumes any legal liability or responsibility for the accuracy, completeness, or usefulness of any information, apparatus, product, or process disclosed, or represents that its use would not infringe privately owned rights. Reference herein to any specific commercial product, process, or service by trade name, trademark, manufacturer, or otherwise, does not necessarily constitute or imply its endorsement, recommendation, or favoring by the United States Government or any agency thereof. The views and opinions of authors expressed herein do not necessarily state or reflect those of the United States Government or any agency thereof. 


\title{
EVALUATION OF CANDIDATE PHOTOMULTIPLIER TUBES FOR THE UPGRADE OF THE CDF END PLUG CALORIMETER
}

\author{
W. KOSKA, S. W. DELCHAMPS, J. FREEMAN, W. KINNEY, D. LEWIS, P. LIMON, \\ J. STRAIT \\ Fermi National Accelerator Laboratory*, P.O. Box 500 \\ Batavia, Mlinois, 60510, USA \\ I. FIORI \\ INFN and Dipartimento di Fisica, University of Bologna, Bologna, Italy \\ M. GALLINARO \\ INFN and Dipartimento di Fisica, University of Padova, Padova, Italy \\ Q. SHEN \\ Department of Physics, Purdue University \\ West Lafayette, Indiana, 47904, USA
}

\begin{abstract}
The Collider Detector at Fermilab is upgrading its end plug calorimeter from a gas detector system to one using scintillating tiles and wavelength shifting fibers. This tile-fiber calorimeter will be read out through 1,824 photomultiplier tubes. The performance requirements of the calorimeter require that the PMTs have good response to light in the $500 \mathrm{~nm}$ region, provide adequate amplification for signals from minimum ionizing particles yet provide linear response for peak anode currents up to $25 \mathrm{~mA}$ at a gain of 50,000 , and fit into the restricted space at the rear of the plugs. This paper will describe the evaluation process used to determine the adequacy of the commercially available PMTs which appeared to meet our performance requirements.
\end{abstract}

\section{Introduction}

The Collider Detector at Fermilab (CDF) ${ }^{1}$ is upgrading its end plug calorimeter from a gas detector system ${ }^{2}$ to one using scintillating tiles read out through wavelength shifting (WLS) fibers ${ }^{3}$. This tile-fiber calorimeter is longitudinally segmented into electromagnetic and hadronic sections which will be read out through 1824 photomultiplier tubes (PMTs). For convenience, we desired a single PMT type be used for both calorimeters even though the inherent difference between electromagnetic and hadronic showers require those PMTs which service the hadronic calorimeter to operate at a gain approximately ten times that of those servicing the EM calorimeter. The overall gain regime runs from $1 \times 10^{4}$ to $1 \times 10^{\circ}$, and the PMTs must respond linearly for input signals ranging from approximately 50 photoelectrons to $1.5 \times 10^{5}$ (EM) and $1.2 \times 10^{4}$ (hadron). The phototubes must also fit into the restricted space at the rear of the plug calorimeter.

These requirements limited the search for an acceptable PMT to those with:

*Work supported by the US Department of Energy. 
1) green-extended photocathodes to provide a good match to the wavelength of light emitted from the WLS fiber, 2) eight or ten stages to provide adequate gain range, and 3) a diameter of less than $20 \mathrm{~mm}$ in order to fit within the limited space but with the photocathode acive area greater than $15 \mathrm{~mm}$ in diameter to contain the fibers from all the layers of a calorimeter tower. Phototubes which appeared to meet most of these requirements were the Burle C83012E (10 stages), the Hamamatsu R4125 (10 stages), the Philips XP1901 (10 stages), the Philips XP1981 (8 stages) and the Thorn EMI D919B (10 stages). We learned, however, that the C83012E used a wire anode which restricted its linear range to less than $1 \mathrm{~mA}$ peak current, which was well below our requirements. We also concentrated on studying the XP1981 over the XP1901 since the vendor suggested that it more closely matched our needs. The candidate PMTs were evaluated with regard to their performance relative to the following characteristics: 1) gain versus voltage, 2) dark current versus voltage 3) relative quantum efficiency, 4) photocathode response, 5) linearity of response to various input signals, and 6) stability. The results of these evaluations will be presented in the following sections.

\section{Determination of Gain and Dark Current versus Voltage}

A PMT's gain was estimated by obtaining its response distribution for 10,000 flashes from a red LED. The gain may be related to the ratio of the mean value of the distribution and the square of the width4. This technique is convenient to use, however it is known to overestimate the actual gain of the PMT since it is affected by each tube's intrinsic pulse height resolution. The pulse height resolution corrections are smallest at highest gain, so we determined the gain using this method once (requiring gain $>10^{5}$ ) and then obtained gains at subsequent voltages using the ratio of the mean values of the PMT's response distributions. Figure 1 displays histograms for several tube types which depict the spread in the required voltages to produce a gain of $1 \times 10^{\circ}$. We received R4125 PMTs from Hamamatsu which had serial numbers prefixed with a CX or ZA and permanently attached bases. A third set of R4125's were tested with voltage divider networks recommended by the vendor (modified type B) for improved linearity at low gains. The Thorn EMI 9078A is the counterpart of the D919B without a green extended photocathode. We would expect it to behave, in most respects not associated with cathode response, in an identical manner to that of the D919B.

The dark current of the PMTs was determined at a sequence of voltages using a Kiethly 486 picoammeter. All tubes met our requirements of less than $5 \mathrm{nA}$ dark current at a gain of $5 \times 10^{5}$.

\section{Relative Quantum Effliency}

The relative quantum efficiency of candidate PMTs was determined by measuring their response to light pulses produced by a tile-fiber combination typical of those to be used in the calorimeter, in order to insure that the correct light spec- 


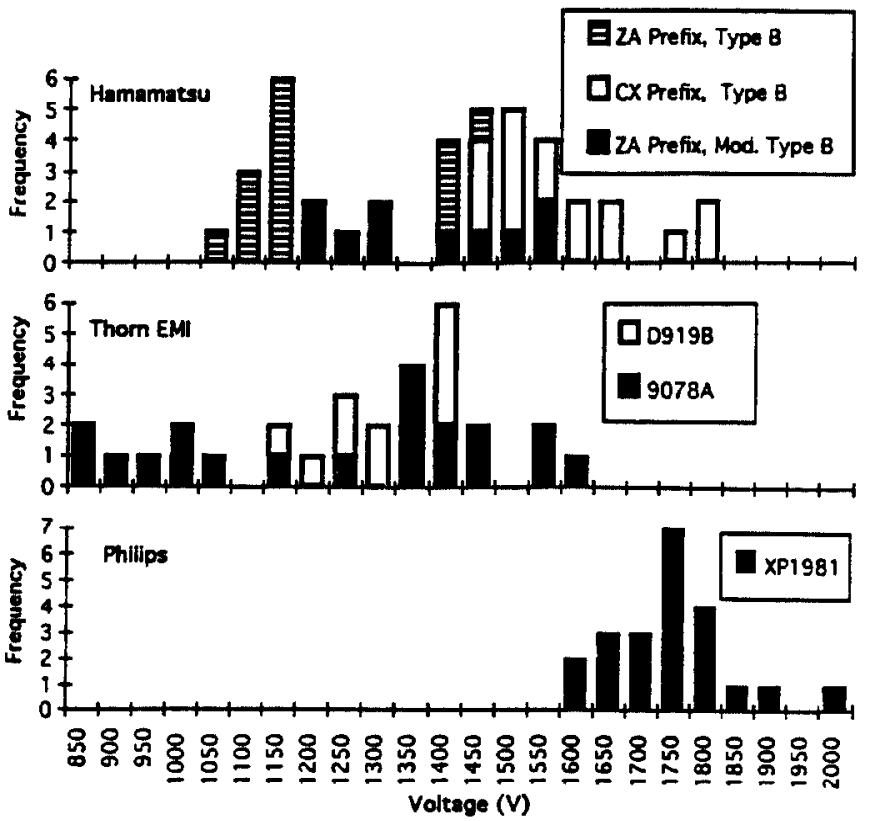

Figure 1: Voltage distributions for PMTs operating at a gain of $1 \times 10^{\circ}$.

trum was being used. The light from the fiber was coupled to the PMT through a $10.5 \mathrm{~mm} \times 10.5 \mathrm{~mm} \times 45 \mathrm{~mm}$ light mixer similar to that which will be used in the calorimeter. The tile was placed within a scintillating paddle telescope and the average number of photoelectrons produced by the transit of a $\beta$ particle from a ${ }^{100} \mathrm{Ru}$ source was determined from the number of pedestal counts, assuming Poisson statistics. The results of these measurements are shown in Figure 2. The green extended Philips and Thorn EMI tubes exhibited comparable efficiencies, while the Hamamatsu R4125's appeared to be about $20 \%$ more efficient on average.

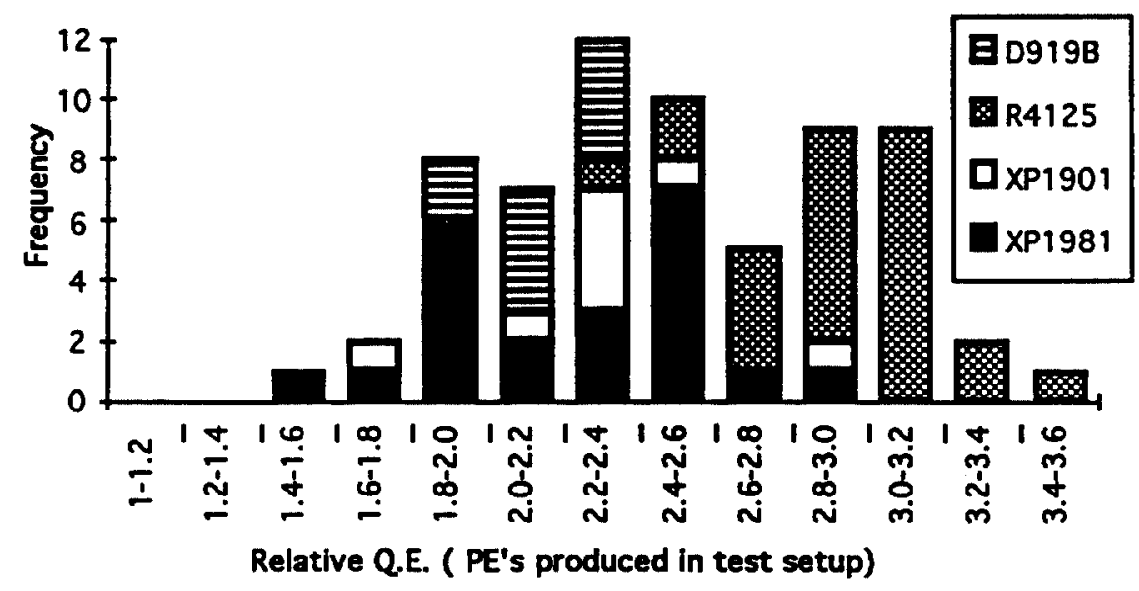

Figure 2: Relative quantum efficiency of PMTs with respect to light produced by WLS fiber. 

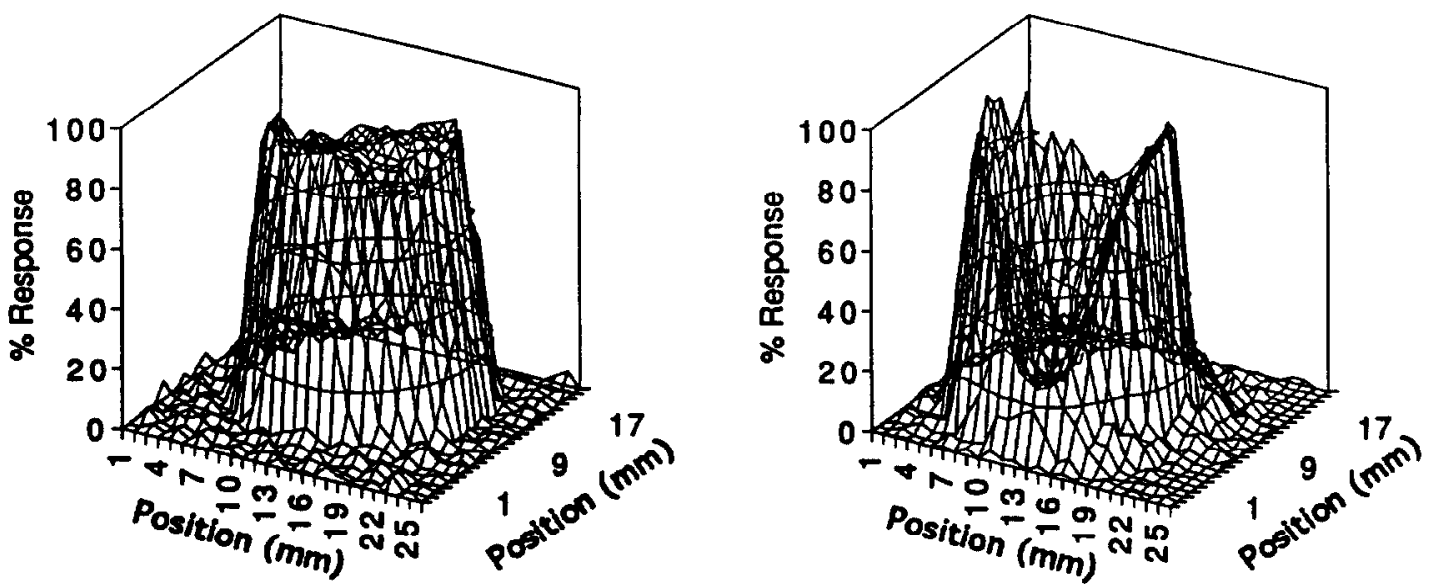

Figure 3: Photocathode uniformity of an R4125 and an XP1981 PMT. The vertical scale is the per cent response relative to the maximum response observed.

\section{Photocathode Uniformity}

A map of the photocathode response of at least two PMTs of the most promising candidates were made by scanning a $1 \mathrm{~mm}$ fiber across the face of the tube in $1 \mathrm{~mm}$ steps $^{5}$. At each position the mean response of the tube was determined. Sharp variations in the response could be indicative of manufacturing problems. Figure 3 compares the response of a Hamamatsu R4125 PMT and a Philips XP1981 PMT. The R4125 appears to be uniform to within 20\% over a central region approximately $10 \mathrm{~mm}$ in diameter. The XP1981 showed a region of reduced sensitivity near the center of its active area, relative to the perimeter, on both tubes scanned.

\section{Linearity of Response}

The PMTs are required to respond in a linear manner for signals ranging from those produced by minimum ionizing particles to those produced by $400 \mathrm{GeV}$ showers. The value of the current at the peak of the anode pulse at which response deviated from linearity by more than $2 \%$ was determined for candidate PMTs by measuring their response to light flashes produced by a UV laser shining on a scintillator and delivered to the tubes by way of a wavelength shifting fiber which viewed the scintillator. The laser flashes were normalized on a pulse by pulse basis using a PIN diode to compensate for variations in laser output. Measurements were made at gains of $10^{4}, 5 \times 10^{4}, 10^{8}$ and $5 \times 10^{5}$ in order to span the range over which the tubes are expected to operate.

To measure linearity, the PIN diode and PMT responses are recorded for several thousand flashes of the laser while the laser light passes through a rotating filter 


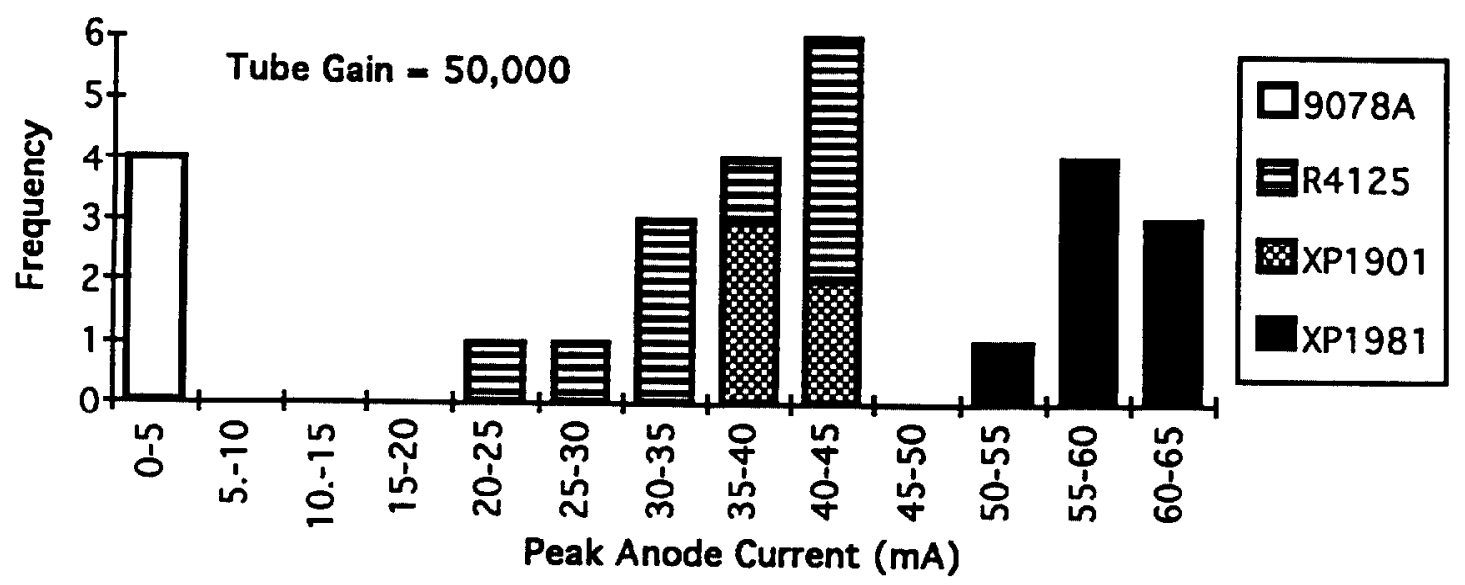

Figure 4: The peak current at which PMT response first deviated by $2 \%$ from linearity.

wheel with optical density logarithmically distributed between 0.1 and 2.0 ( $\sim 90 \%$ to $1 \%$ transmission). A fitting algorithm is applied to the data to determine the peak anode current at which the PMT response falls below $2 \%$ of linearity. Figure 4 is a histogram of the peak currents at which response first deviated by greater than $2 \%$ from linearity for tubes operating at a gain of 50,000 . The Thorn EMI tubes fall below our desired maximum peak current of $25 \mathrm{~mA}$ at this gain. All tubes were tested using the manufacturer's recommended voltage divider configuration for good linearity while maintaining adequate gain range.

\section{Stability}

Stability of PMT response with regard to three criteria were evaluated: 1) stability versus time, 2) stability versus turning high voltage off and on, and 3) stability versus average anode current. PMT response was determined at 15 minute intervals over a period of several days. The tube was excited using a laser source as with the linearity measurement. We were interested in turn-on effects that might be present, as well as long term drifts. The effects of cycling the PMT voltage was determined by comparing the response of tubes before and after cycling. We were interested in this since during the setup of colliding beams at the Fermilab Tevatron, beam losses may make it necessary to turn down or off the high voltages of PMTs in the plug calorimeter. Variation in the response of the PMTs to pulsed light in the presence of a steady state background light was also evaluated. This background was provided by a tungsten lamp in our tests but would be provided by minimum bias events during operation of the detector. Minimal variation is desired to make calibration easier.

Our criteria for acceptable performance with respect to stability were: 1 ) less than $\pm 2 \%$ deviation in response over any 48 hour period, 2) a return to within $\pm 3 \%$ of previous response value within 10 minutes of the tube voltage being turned off, 
then on, 3) less than $\pm 5 \%$ variation in PMT response to a pulsed signal for average anode currents up to $1 \mu \mathrm{A}$ from the dark current floor. Although some PMTs failed to meet these criteria, there were not enough of any one type to merit disqualification of a vendor. Tests similar to those listed here will be used during acceptance testing of the production tubes to reject those tubes exhibiting poor performance.

\section{Conclusions}

We have evaluated five different photomultiplier tubes which met our initial requirements with regard to size and sensitivity to the relevant spectrum of light from WLS fiber. Of these, the Burle C83012E was not designed to operate at high peak currents. The remaining tubes could meet our gain and dark current requirements. The Hamamatsu $\mathbf{R} 4125$ appeared to have approximately $20 \%$ higher quantum efficiency than the other tubes and had a more uniform response over its active area. The Thorn EMI D919B tube had significantly less linear range than the R4125 or the XP1981. All four tube types performed comparably with respect to stability criteria. The CDF end plug upgrade project will use the R4125 PMT.

\section{Acknowledgements}

The authors would like to thank the members of the Fermilab Accelerator and Detector R\&D group whose work made possible the measurements presented here. We would also like to thank Ms. Lauren Curry for her help with the preparation of this paper.

\section{References}

1. F. Abe et al., Nucl. Instr. and Meth. A271 (1982) 2015.

2. Y. Fukui et al., Nucl. Instr. and Meth. A267 (1988) 280.

3. G. Apollinari, P. de Barbaro, M. Mishina, CDF Calorimeter Upgrade Project to be published in the Proc. of the 4th Int. Conf. on Calorimetry in High Energy Physics, 1993

4. T. Devlin et al., Nucl. Instr. and Meth. A26B (1988) 24.

5. B. H. Tannenbaum, New Methods in Optical Fiber Preparation for Scintillating Tile Calorimetry, Master's Thesis, Michigan State University, (1993) 